ARTIGO ORIGINAL

\title{
Descontaminação em processo de contracorrente de madeira tratada com CCA e tratamento do efluente gerado por eletrorrecuperação e precipitação
}

\author{
Counter-current process for cca-treated wood decontamination and treatment of \\ effluent generated by electric recovery and precipitation
}

\author{
Heldiane Souza dos Santos ${ }^{1}$ (), Suzana Frighetto Ferrarini ${ }^{1}$ (1), Marçal José Rodrigues Pires $^{1}$ (D),

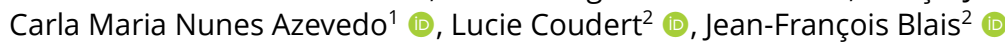 \\ ${ }^{1}$ Pontifícia Universidade Católica do Rio Grande do Sul - PUCRS, Porto Alegre, RS, Brasil \\ ${ }^{2}$ Eau Terre et Environnement, Institut Nacional de la Recherche Scientifique - INRS, Quebec, QC, CA
}

\begin{abstract}
Como citar: Santos, H. S., Ferrarini, S. F., Pires, M. J. R., Azevedo, C. M. N, Coudert, L., \& Blais, J.-F. (2020). Descontaminação em processo de contra-corrente de madeira tratada com CCA e tratamento do efluente gerado por eletrorrecuperação e precipitação. Scientia Forestalis, 48(127), e3234. https://doi.org/10.18671/scifor.v48n127.08
\end{abstract}

\begin{abstract}
Resumo
A utilização do preservante CCA (Arseniato de Cobre Cromatado) para preservação de madeira tem sido uma alternativa para aumentar sua durabilidade, porém gerando grande volume de efluentes, tanto nas usinas de preservação como em processos de descontaminação da madeira tratada. Em função da presença de elevadas concentrações de elementos tóxicos ( $\mathrm{Cu}, \mathrm{Cr}$ e As) esses efluentes devem ser tratados antes de serem descartados. O objetivo deste trabalho foi desenvolver o método em contracorrente para a descontaminação da madeira tratada com CCA, em que se avaliou a reutilização dos efluentes e das águas de lavagem para cada ciclo de extração. Foram estudados ainda a precipitação total dos elementos $\mathrm{Cu}, \mathrm{Cr}$ e As e a eletrorrecuperação de Cu para o primeiro extrato do processo de contracorrente (L1). Foram obtidos percentuais acima de $98,5 \%$ de remoção total dos elementos por precipitação. Cerca de $91 \%$ de Cu foi recuperado do efluente quando se aplicou uma corrente de $5 \mathrm{~A}$ durante $90 \mathrm{~min}$. Os resultados indicaram que a utilização do processo em contracorrente para descontaminação ácida da madeira tratada com CCA mostrou ser eficiente bem como os tratamentos propostos para os efluentes, obtendo-se um efluente adequado para reutilização ou descarte de acordo com os padrões da legislação ambiental ao final dos processos.
\end{abstract}

Palavras-chave: Elementos tóxicos; Cu; Cr; As; Recuperação.

\section{Abstract}

The preservative Chromate Copper Arsenate (CCA) has been used as an alternative for wood preservation to increase its durability, generating a large volume of effluents at treatment plants and in during the decontamination of treated wood. Due to the presence of high concentrations of toxic elements ( $\mathrm{Cu}, \mathrm{Cr}$ and $\mathrm{As})$ these effluents must be treated before being disposed of. Thus, the aim of this study was to develop an effective wood decontamination method based on the counter-current process, reusing the effluents and rinsing water in each extraction cycle. Additionally, total precipitation of the 3 elements and electric recovery of $\mathrm{Cu}$ for the extract of the counter-current process were also studied. Removal of the elements by precipitation produced percentages higher

Fonte de financiamento: Conselho Nacional de Pesquisa - CNPq (Proc. Nºs 309623/2012-0 e 312323/2015-8). Fundação de Amparo à Pesquisa do Estado do RS - FAPERGS (Proc. № 11/1403-8). Comissão de Aperfeiçoamento de Pessoal do Nível Superior — CAPES (Proc. PDSE n 9108/11-4).

Conflito de interesse: Nada a declarar.

Autor correspondente: heldianedossantos@gmail.com

Recebido: 29 dezembro 2018.

Aceito: 28 agosto 2019.

Editor: Francides Gomes Silva Júnior.

(c) (i) Este é um artigo publicado em acesso aberto (Open Access) sob a licença Creative Commons Attribution, que permite uso, distribuição e reprodução c) em qualquer meio, sem restrições desde que o trabalho original seja corretamente citado. 
than $98.5 \%$. About $91 \%$ of Cu was recovered from the effluent when a current of 5 A was applied for $90 \mathrm{~min}$. The results indicated that the counter-current process was efficient in acid decontamination of CCA-treated wood and the proposed treatments produced an effluent suitable for reuse or disposal, in accordance with environmental legislation.

Keywords: Toxic elements; Cu; Cr; As; Recovery.

\section{INTRODUÇÃO}

No Brasil, cerca de 1,5 milhões de $\mathrm{m}^{3}$ de madeira tratada são comercializados por ano, sendo que $90 \%$ deste volume correspondem ao eucalipto, espécie comumente usada para reflorestamento, seguida pelo pinus (Vidal et al., 2015; Associação Brasileira de Produtores de Florestas Plantadas, 2012; Associação Brasileira dos Preservadores de Madeira, 2016). A maior parte da madeira é tratada com o preservante Arseniato de Cobre Cromatado (CCA) e destinase à produção de postes, moirões, dormentes e diversas peças utilizadas em linhas de distribuição de energia elétrica e telefonia. O consumo de madeira tratada vem aumentando gradativamente no país, apesar de ainda ser pequeno, se comparado ao consumo, por exemplo, nos Estados Unidos, Canadá e Europa (Helsen \& Vandenbulck, 2005; Lombardi, 2010).

Devido à elevada toxicidade de As e $\mathrm{Cr}$ e a possibilidade de lixiviação desses elementos durante a utilização, existem várias restrições quanto ao uso de produtos contendo esse tipo de tratamento preservante para fins residenciais. Nos EUA, Suécia e Dinamarca, esta restrição foi imposta já no ano de 2004 (Ferrarini et al., 2012, 2015; Townsend et al., 2005; Zagury et al., 2008). Apesar dessas restrições, o volume de madeira tratada com CCA consumido no mundo inteiro é relativamente alto devido à vasta gama de aplicações. Com o final de vida útil desses materiais, é previsto no futuro um grande aumento no volume de resíduos contendo esses elementos tóxicos (Vidal et al., 2015).

A descontaminação química da madeira tratada com CCA ao término de sua vida útil é uma alternativa para reduzir o impacto ambiental ocasionado pela presença dos elementos de elevada periculosidade, As e Cr. Desse modo, a remoção completa desses elementos, a partir dos resíduos de madeira, torna-se necessária em termos de segurança ambiental e da saúde humana (Chang et al., 2013). Dentre as metodologias utilizadas para remoção dos componentes presentes nos resíduos da madeira tratada com CCA, a descontaminação ácida destaca-se devido ao baixo custo dos reagentes (ácidos inorgânicos) utilizados e a eficiência no processo de remoção (>85\%) (Ferrarini, 2012; Janin et al., 2009a; Santos, 2014). Ao submeter esses resíduos aos processos de extração ácida pode ocorrer a reversão das reações de fixação, por meio da conversão dos elementos do CCA para suas formas solúveis em água (Clausen et al., 2001). Infelizmente são escassos os estudos sobre o reuso desses efluentes (Janin et al., 2012a) e, no nosso conhecimento, nenhum trabalho foi reportado no país sobre esse tema.

Salienta-se que, após um processo de extração ácida, o produto sólido resultante (madeira descontaminada), pode ser utilizado como matéria-prima para a produção de novos produtos, tais como laminados, painéis e materiais compósitos (Cooper et al., 2001), fonte de carbono na compostagem para fins agrícolas ou residenciais (Banegas et al., 2007; De Guardia et al., 2007) ou, como fonte de energia térmica (Janin et al., 2009a). Entretanto, os processos de descontaminação levam à geração de efluentes complexos, contendo além de elementos tóxicos, teores elevados de matéria orgânica e baixos valores de pH (Santos et al., 2018). Dessa forma, se faz necessário o desenvolvimento de tecnologias sustentáveis que minimizem o lançamento de efluentes, atendendo os padrões previstos na legislação, por meio do seu reuso. Dentre as técnicas utilizadas na remoção dos metais contaminantes a precipitação química é a mais utilizada em função principalmente de sua eficiência, baixo custo e simplicidade (Blais et al., 2008). Entretanto, esse processo gera grande quantidade de lodo, que deve ser tratado e disposto adequadamente, não permitindo a recuperação de elementos de interesse. 
Nesse contexto, o uso de um processo híbrido que associe a precipitação dos elementos de interesse com a eletrorrecuperação do cobre (Britto-Costa \& Ruotolo, 2015) tem sido proposto (Janin et al., 2009b).

Com base no que foi exposto, esse trabalho visa em um primeiro momento desenvolver o método em contracorrente pelo processo de extração ácida com $\mathrm{H}_{2} \mathrm{SO}_{4}$, a fim de garantir uma efetiva descontaminação da madeira tratada com CCA. Para diminuir custos e aumentar a eficiência do processo de descontaminação, os efluentes resultantes e as águas de lavagens empregadas durante os ciclos, foram reutilizados nos ciclos seguintes. Em um segundo momento, foram avaliados dois diferentes processos de recuperação/tratamento para o efluente obtido: a precipitação total dos elementos $\mathrm{Cu}, \mathrm{Cr}$ e As e a eletrorrecuperação de Cu para o primeiro extrato do processo de contracorrente (L1).

\section{MATERIAIS E MÉTODOS}

\section{Experimento de descontaminação da madeira em contracorrente}

Para a realização do experimento foi utilizada uma mistura de amostras de postes de madeira retirados de serviço após 14 anos (15 postes). Esses postes foram fabricados com diferentes espécies do gênero Eucalyptus sp, denominada Amostra Geral (AG), doados pela Companhia Gaúcha de Distribuição de Energia AES Sul, provenientes da cidade de Canoas/RS, Brasil. Em todos os testes foram utilizados o cerne e o alburno da madeira. A escolha da utilização do cerne e do alburno para realização dos testes baseiase nos estudos desenvolvidos por Ferrarini (2012), onde os resíduos de madeira tratada foram submetidos ao teste de lixiviação, de acordo com a NBR 10005 (Associação Brasileira de Normas Técnicas, 2004b), utilizando somente o alburno e uma mistura de cerne + alburno, e, em ambos os casos, o resíduo de madeira tratada com CCA foi considerado perigoso (NBR 10004: Associação Brasileira de Normas Técnicas, 2004a). A redução de tamanho das amostras dos postes de madeira com cerca de $12 \mathrm{~m}$ de altura, foi feita com a redução de tamanho para torretes (cerca de $30 \mathrm{~cm}$ ) e após discos com $5 \mathrm{~cm}$. Para os testes de descontaminação da madeira tratada, as amostras foram reduzidas apenas com o auxílio de formão e martelo e passadas em peneira com abertura de malha $\leq 9,5 \mathrm{~mm}$.

A descontaminação da madeira tratada com CCA pelo processo em contracorrente foi realizada em triplicata, com sete ciclos fechados de descontaminação (C1-C7), totalizando 21 extrações (Figura 1). Apenas para o primeiro ciclo (C1) foi utilizada solução de extração $\left(0,1 \mathrm{~mol} \mathrm{~L}^{-1} \mathrm{H}_{2} \mathrm{SO}_{4}\right)$ e água de lavagem nova. Cada ciclo do processo de descontaminação contém 3 etapas de extração, com 2 h de duração, denominadas: lixiviação 1 (L1), lixiviação 2 (L2) e lixiviação 3 (L3) e 3 etapas de lavagem (R1, R2 e R3). O lixiviado L2 foi reutilizado em L1, e o lixiviado L3 em L2 do próximo ciclo. A partir do segundo ciclo (C2), a água de lavagem foi reutilizada para a lavagem do ciclo seguinte, tendo seu volume reajustado para $2 \mathrm{~L}$. Para cada ciclo (C2-C7), na última etapa de lavagem (R3), foram utilizados $2 \mathrm{~L}$ de água potável. A Figura 1 também apresenta o esquema de reaproveitamento dos efluentes resultantes do $1^{\circ}$ ciclo de extração para o $2^{\circ}$ ciclo durante a descontaminação da madeira, após precipitação alcalina, descrita no próximo subitem. Nos ciclos seguintes $\left(3^{\circ}\right.$ ao $\left.7^{\circ}\right)$, os efluentes seguiram a mesma sequência de reaproveitamento. A reutilização do efluente tratado não só permite a redução da quantidade de água e de efluentes do processo, como também reduz os custos de operação. Essa operação foi repetida por mais seis vezes para completar os sete ciclos do experimento. 


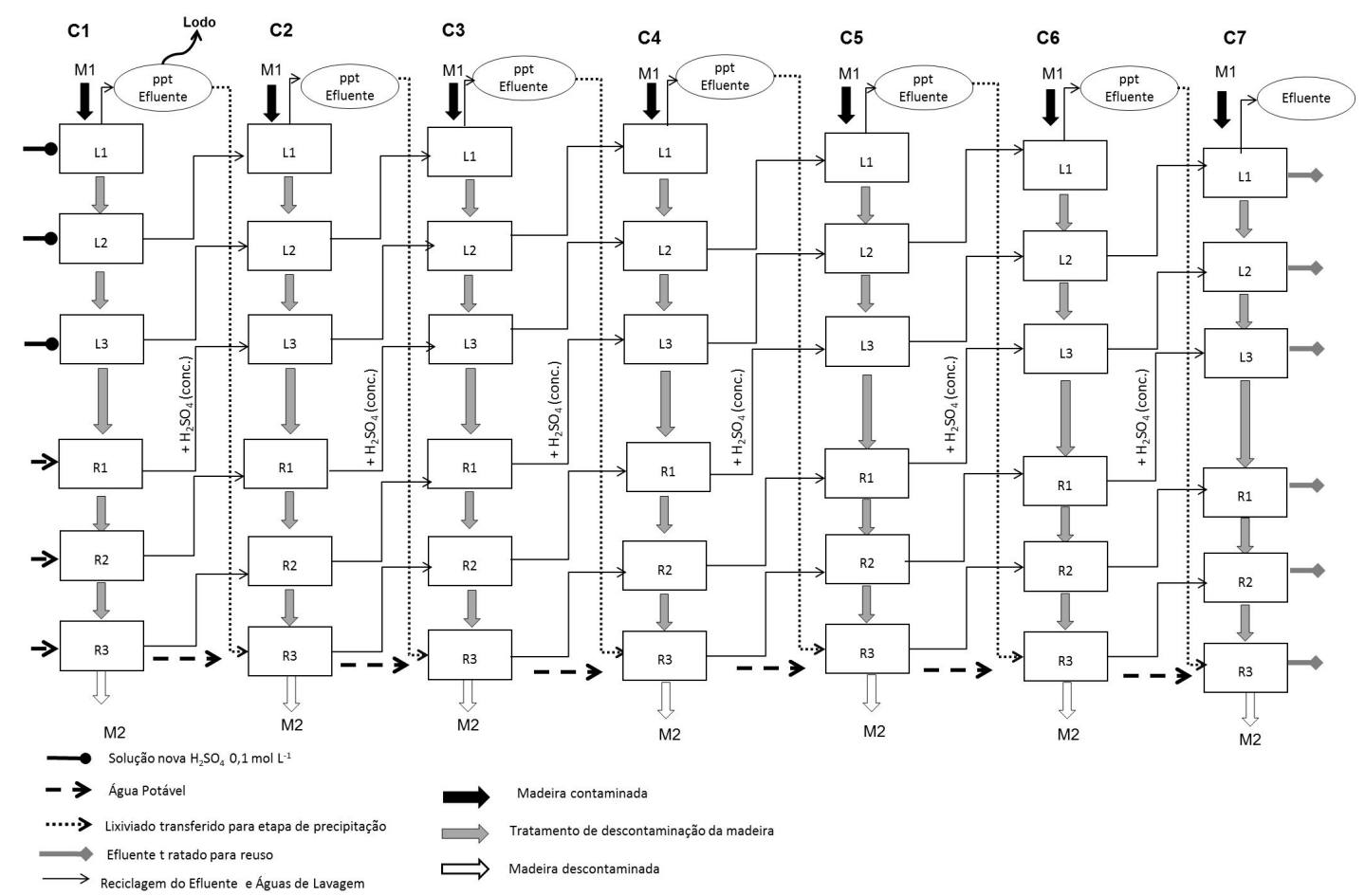

Figura 1. Esquema do processo de descontaminação da madeira pelo processo em contracorrente com o reaproveitamento do efluente.

O processo de descontaminação utilizado foi previamente otimizado por Ferrarini et al. (2016) onde em cada ciclo foram utilizados $300 \mathrm{~g}$ de amostra de Eucalyptus sp (AG) e $2 \mathrm{~L}$ de extrator $\left(\mathrm{H}_{2} \mathrm{SO}_{4} 0,1 \mathrm{~mol} \mathrm{~L}^{-1}\right)$ colocados em contato por $2 \mathrm{~h}$, em um copo de béquer de vidro com capacidade para $5 \mathrm{~L}$. A mistura foi, então, aquecida em chapa elétrica (Corning) a $75^{\circ} \mathrm{C} \mathrm{e}$ mantida sob agitação mecânica (Caframo BDC 3030) a 300 rpm. A descontaminação foi realizada em 3 ciclos de $2 \mathrm{~h}$, seguidos por 3 etapas de lavagem com $2 \mathrm{~L}$ de água potável à temperatura ambiente $\left(25^{\circ} \mathrm{C}\right)$, de 7 minutos cada. A separação sólido/líquido foi realizada utilizando bomba de vácuo e os extratos e as águas de lavagem resultantes foram filtrados em membranas de fibra de vidro 1,5 $\mu$ m (Whatman 934-AH). Em todas as etapas e ciclos de descontaminação da madeira foram retiradas amostras para a quantificação dos elementos $\mathrm{Cu}, \mathrm{Cr}$ e As por Espectrometria de Emissão Atômica com Plasma Indutivamente Acoplado (ICP AES) (Vista AX CCO Varian, USA), medidas de pH e carbono orgânico dissolvido - COD (TOC-5000A Shimadzu, Japão).

\section{Tratamento do Efluente}

\section{Precipitação alcalina dos elementos}

Para a otimização do processo de precipitação dos elementos foi utilizado o efluente obtido a partir do processo de descontaminação da madeira tratada (L1). Os experimentos foram realizados em triplicata, em béquer de $100 \mathrm{~mL}$, com agitação magnética (100 rpm). Para tanto, os lixiviados foram tratados com diferentes concentrações de solução de cloreto férrico $\left(131,393,655,917,1.180\right.$ e $1.572 \mathrm{mg} \mathrm{L}^{-1}$ de $\left.\mathrm{Fe}^{3+}\right)$. O pH da solução foi ajustado para $7 \mathrm{com}$ soluções de $\mathrm{NaOH}$ ou $\mathrm{Ca}(\mathrm{OH})_{2} 100 \mathrm{~g} \mathrm{~L}^{-1}$. Após a definição da melhor concentração de $\mathrm{FeCl}_{3}, \mathrm{O}$ experimento foi realizado em diferentes valores de $\mathrm{pH}(2$ - 9) afim de obter a melhor remoção dos elementos. O sobrenadante foi recolhido e filtrado em membrana de fibra de vidro $(1,5 \mu \mathrm{m})$ para análise dos elementos residuais por ICP AES.

Após a otimização do processo e definição das melhores condições $\left(1.180 \mathrm{mg} \mathrm{L}^{-1} \mathrm{Fe}^{+3} \mathrm{e}\right.$ $\mathrm{pH}$ 7), os ensaios de precipitação foram realizados utilizando soluções de $\mathrm{NaOH}$ ou $\mathrm{Ca}(\mathrm{OH})_{2}$ $100 \mathrm{~g} \mathrm{~L}^{-1}$. Volumes das soluções de $\mathrm{NaOH}$ ou $\mathrm{Ca}(\mathrm{OH})_{2}$ e $\mathrm{FeCl}_{3}$ foram utilizados para a 
precipitação até atingir o pH desejado. Após a adição dos reagentes, a agitação foi mantida a 100 rpm por 60 min para completa estabilização e a mistura foi colocada em repouso por uma noite para a completa decantação. $O$ agente precipitante foi o cloreto férrico devido a sua simplicidade, versatilidade, seletividade e baixo custo (Santos et al., 2018). O processo consiste em ajustar apenas o pH e a dosagem do sal de ferro para remoção das espécies metálicas. Devido à baixa solubilidade dos hidróxidos férricos formados, eles podem agir em ampla faixa de $\mathrm{pH}$. O cloreto férrico produz bons flocos na faixa de pH entre 5,0 a 11,0 (Spinelli, 2001). Adicionalmente, os íons férricos também reagem diretamente com o As, presente na forma de $\mathrm{AsO}_{4}{ }^{3-}$ nessas condições (Janin et al., 2009a), gerando arseniato de ferro insolúvel. Dessa forma, a presença de íons férricos é necessária para a remoção eficiente desse elemento.

$\mathrm{Na}$ última etapa, o sobrenadante foi separado com auxílio de uma bomba peristáltica (Masterflex L/S77200-52-ColeParmer) para posterior reutilização e, o lodo separado para correto descarte. Foram também testados dois floculantes poliméricos (sintético FX NS2, natural Acquapol WW) visando melhorar o desempenho do processo de separação pela remoção de flocos menores e a redução do lodo formado (Jekel \& Amy, 2006). Porém, os resultados não foram satisfatórios ocorrendo um aumento na concentração do As e uma redução pouco significativa do volume de lodo. Em função disso, os demais testes foram feitos sem adição desses floculantes auxiliares. Em todas as etapas foram retiradas alíquotas do sobrenadante para análise dos elementos por ICP AES, COD e pH.

\section{Eletrorrecuperação de cobre}

Os ensaios de eletrorrecuperação de cobre foram conduzidos em regime de batelada utilizando uma célula eletrolítica de acrílico, com dimensões de $12 \mathrm{~cm}$ (largura) $\times 12 \mathrm{~cm}$ (comprimento) $\times 19 \mathrm{~cm}$ (profundidade). O conjunto de eletrodos com área de $172,5 \mathrm{~cm}^{2}$ consistiram de 8 placas paralelas, 4 cátodos e 4 ânodos, posicionados a $1 \mathrm{~cm}$ de distância entre si e submersos em $2 \mathrm{~L}$ de eletrólito. Os cátodos (placas de aço inoxidável AISI 302) foram alternados com ânodos (placas perfuradas de aço inoxidável AISI 302). Os eletrodos foram instalados sobre uma placa de acrílico perfurada que foi suportada a $1,5 \mathrm{~cm}$ do fundo da célula. Para agitação magnética da solução, foi utilizada barra revestida de Teflon. Inicialmente foram utilizados $2 \mathrm{~L}$ de uma solução sintética de $\mathrm{CuSO}_{4} .5 \mathrm{H}_{2} \mathrm{O}$ com concentração de $60 \mathrm{mg} \mathrm{L}^{-1}$ para otimização do tempo e corrente a serem utilizados e, após foi utilizado $2 \mathrm{~L}$ do efluente contendo duas diferentes concentrações de cobre (62 e $166 \mathrm{mg} \mathrm{L}^{-1} \mathrm{de} \mathrm{Cu}^{2+}$ ), obtidos no processo de descontaminação da madeira tratada. Amostras de $10 \mathrm{~mL}$ foram coletadas a cada 15 minutos para determinação da concentração de $\mathrm{Cu}$ da solução sintética e de $\mathrm{Cu}, \mathrm{Cr}$ e As das amostras de efluentes utilizadas. A quantificação foi realizada por ICP AES. Os conjuntos de ânodo e cátodo foram ligados na fonte de alimentação simétrica (Minipa MPC 3003 D). Foram utilizadas correntes de 1,0; 2,5; 5,0; 7,5 e 10,0 A, mantidas constantes, à temperatura ambiente, para cada ensaio com um tempo total de 90 min.

\section{RESULTADOS E DISCUSSÃO}

\section{Caracterização e descontaminação em contracorrente da madeira tratada}

A madeira tratada com CCA utilizada apresentou uma umidade de $12,1 \%$ e uma concentração inicial média de $\mathrm{Cu}, \mathrm{Cr}$ e As de $1.350 \pm 85 \mathrm{mg} \mathrm{kg}^{-1}, 2.070 \pm 160 \mathrm{mg} \mathrm{kg}^{-1} \mathrm{e}$ $1.800 \pm 127 \mathrm{mg} \mathrm{kg}^{-1}$, respectivamente (Tabela.1). Essas concentrações estão abaixo da gama reportada na literatura para postes de eucalipto tratados com CCA em serviço (Ferrarini, 2012; Ferrarini et al., 2016). Esse comportamento está associado ao tipo de amostra utilizada neste estudo, resíduos de postes de madeira da espécie Eucalyptus $s p$ retirados de serviço após 14 anos. As menores concentrações podem ser devidas a processos de lixiviação no período em que estiveram instalados na rede de distribuição de energia elétrica. 
A Tabela 1, além de apresentar a concentração inicial dos elementos na madeira tratada com CCA, também mostra a concentração dos mesmos nas 7 amostras de madeira descontaminada (denominação M2, Figura 1), com o percentual médio de remoção dos elementos obtidos após as 3 etapas de descontaminação e 3 etapas de lavagem nos ciclos de 1 a 7. As maiores concentrações residuais médias considerando os três testes realizados foram observadas para o $\mathrm{Cr}\left(312 \pm 26 \mathrm{mg} \mathrm{kg}^{-1}\right)$, seguido do As $\left(122 \pm 16 \mathrm{mg} \mathrm{kg}^{-1}\right)$ e $\mathrm{Cu}(76 \pm 16 \mathrm{mg}$ $\left.\mathrm{kg}^{-1}\right)$. A maior dificuldade de remoção do $\mathrm{Cr}$ está relacionada a sua propriedade de fixação, formando complexos insolúveis por meio de ligações químicas com os componentes da madeira (Jang et al., 2002).

Tabela 1. Concentrações dos elementos na madeira tratada com CCA antes (inicial) e após (residual) descontaminação pelo processo de contracorrente, e percentuais de remoção dos elementos para o $1^{\circ}$ teste.

\begin{tabular}{cccc}
\hline \multicolumn{3}{c}{ Concentração $\left(\mathbf{m g ~ \mathbf { ~ k g } ^ { - 1 } )}\right.$} \\
\hline Inicial & $\mathbf{C u}$ & $\mathbf{C r}$ & As \\
Ciclos & $1.350 \pm 85$ & $2.070 \pm 160$ & $1.800 \pm 127$ \\
C1 & & $1^{\circ}$ Teste \\
C2 & $23 \pm 2$ & $224 \pm 10$ & $62 \pm 4$ \\
C3 & $74 \pm 2$ & $364 \pm 21$ & $142 \pm 3$ \\
C4 & $36 \pm 3$ & $269 \pm 24$ & $87 \pm 14$ \\
C5 & $23 \pm 1$ & $326 \pm 12$ & $104 \pm 2$ \\
C6 & $25 \pm 0$ & $245 \pm 15$ & $91 \pm 0$ \\
C7 & $24 \pm 1$ & $277 \pm 19$ & $91 \pm 6$ \\
\% remoção & $26 \pm 1$ & $238 \pm 17$ & $89 \pm 3$ \\
\hline
\end{tabular}

Foram observadas variações nas concentrações residuais entre os três testes em contracorrente, em especial para o Cr. Observa-se um aumento na concentração residual até o $3^{\circ}$ ciclo seguido de uma queda para valores semelhantes ao $1^{\circ}$ ciclo, em especial para o $\mathrm{Cr}$, no $5^{\circ}$ ciclo. Após, se verifica um novo aumento na concentração para os três elementos nos dois últimos ciclos, com maior variação para os três elementos no $7^{\circ}$ ciclo. Esse comportamento pode estar relacionado em parte ao aumento das concentrações dos elementos e da matéria orgânica nos efluentes reusados, ao longo dos ciclos, conforme dados apresentados no próximo item. Soluções extratoras já contendo os elementos de interesse podem diminuir a eficiência da descontaminação. Entretanto, cabe salientar que não foi verificada diminuição significativa da eficiência de remoção ao logo do processo. Os percentuais de remoção médios considerando os três testes foram de $85 \pm 4 \%$ para $\mathrm{Cr}, 93 \pm 2 \%$ para As e $94 \pm 3 \%$ para Cu.

Esses resultados indicam que a descontaminação da madeira tratada com CCA foi eficiente para as espécies de Eucalyptus sp. Janin et al. (2012b), empregando outra espécie de madeira tratada (Red pine), também reportaram comportamento similar dos elementos ao longo dos ciclos de descontaminação.

\section{Caracterização dos efluentes gerados no processo em contracorrente}

A caracterização dos efluentes gerados no processo descontaminação foi realizada medindo-se as concentrações dos elementos $\mathrm{Cu}, \mathrm{Cr}$ e As além de pH e COD. A Figura 2A apresenta os resultados das concentrações médias dos elementos nos 3 testes em contracorrente realizados para as etapas de descontaminação (extratos L1, L2 e L3) e a Figura 2B para etapas de lavagem (R1, R2 e R3). Observa-se as concentrações elevadas e semelhantes para os três elementos ( $\mathrm{Cu} 182 \pm 24$, Cr $210 \pm 10$, As $214 \pm 6 \mathrm{mg} \mathrm{L}^{-1}$ ) no extrato oriundo na primeira etapa de descontaminação (L1). Cabe destacar a pequena variação entre 
os três testes nessas concentrações (desvio percentual de 3 a 13\%), indicando boa reprodutibilidade. Como esperado, diminuições significativas nessas concentrações foram observadas para a segunda (L2, -67\%) e terceira (L3, - 87\%) etapa de descontaminação. Esses resultados estão em acordo ao esperado em processos de extração sucessivos reportados por Ferrarini et al. (2016). Concentrações bem menores (R1: Cu 6 $\pm 4, \mathrm{Cr} 8 \pm 2$, As $8 \pm 3 \mathrm{mg} \mathrm{L}^{-1}$ ) e decrescentes foram observadas nas etapas consecutivas de lavagens (R2 e R3). Apesar dos baixos teores dos elementos observados nas águas de lavagem, essa etapa é necessária para atingir o nível de descontaminação adequado para o resíduo de madeira ser considerado não perigoso (Ferrarini et al., 2016).

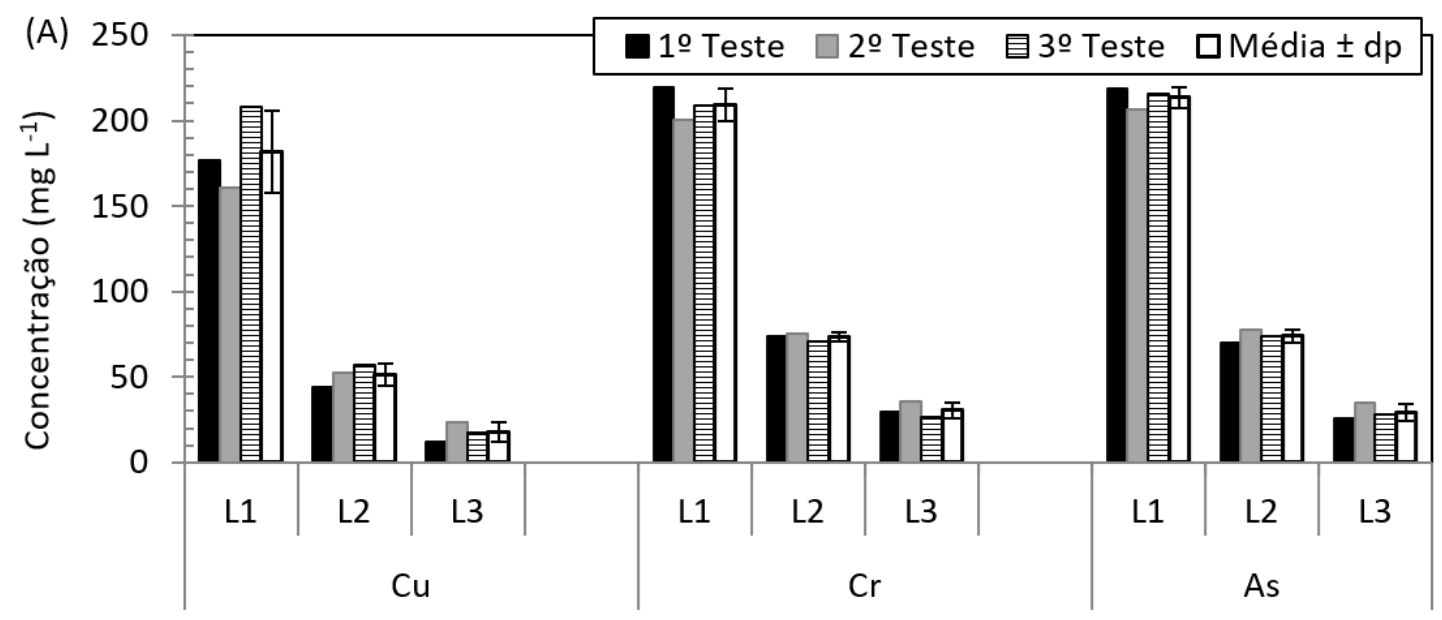

Efluentes / Elementos

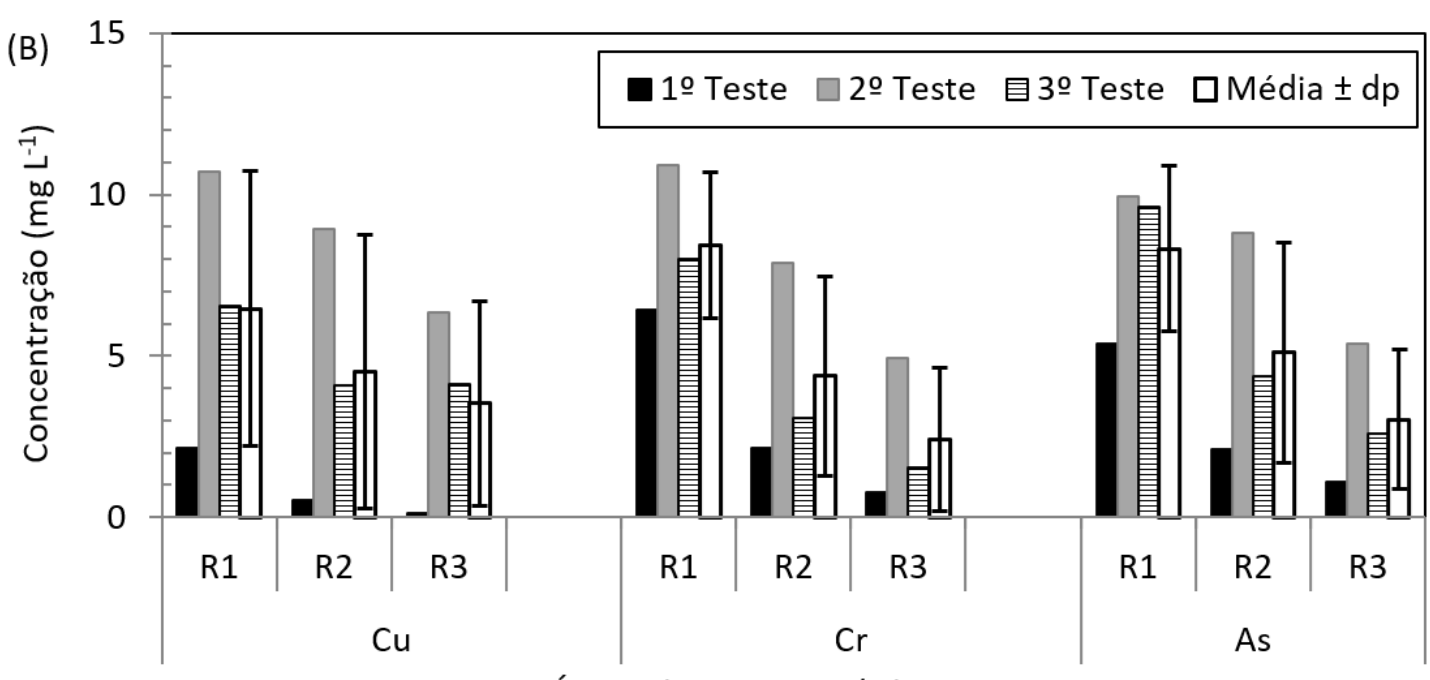

Águas de Lavagens / Elementos

Figura 2. Concentração média dos elementos nos efluentes obtidos pelo processo em contracorrente nas etapas de extração (A) e lavagem (B). Média dos sete ciclos.

Um aumento da matéria orgânica dissolvida, estimada pelo COD, também foi observado, ocorrendo tanto para as 3 etapas de extração (L1: de 766 para $1.944 \mathrm{mg} \mathrm{L}^{-1}$; L2: de 702 para $1.158 \mathrm{mg} \mathrm{L}^{-1}$; L3: de 308 para $769 \mathrm{mg} \mathrm{L}^{-1}$ ) como para as 3 etapas de lavagem (R1: de 36 para $321 \mathrm{mg} \mathrm{L}^{-1} ; \mathrm{R} 2$ : de 20 para $270 \mathrm{mg} \mathrm{L}^{-1} ; \mathrm{R} 3$ : de 39 para $289 \mathrm{mg} \mathrm{L}^{-1}$ ). Um aumento de matéria orgânica na solução ácida de lixiviação pode ser responsável pela redução de $2 \%$ da solubilização de metais (Janin et al., 2011).

Em relação ao pH (resultados não apresentados), houve uma variação de 0,8 a 2,0 para as etapas de extração e de 1,4 a 3,8 para as etapas de lavagem. Como pode ser inferido, os efluentes gerados, principalmente nas etapas de extração possuem caráter ácido, alta 
concentração dos elementos $\mathrm{Cu}, \mathrm{Cr}$ e As e elevada carga orgânica. Dessa forma, não pode ser descartado da maneira como se encontra, sendo necessário passar por um processo de recuperação e/ou tratamento para que os padrões de emissão nacionais (Brasil, 2011) sejam atendidos. Os limites de emissão para os elementos de interesse são $0,5 \mathrm{mg} \mathrm{L}^{-1} ; 1,0 \mathrm{mg} \mathrm{L}^{-1}$; $<1,0 \mathrm{mg} \mathrm{L}^{-1}$ para As total, Cu dissolvido e $\mathrm{Cr}$ trivalente, respectivamente, e o $\mathrm{pH}$ deve estar entre 5,0 e 9,0. Cabe salientar que mesmo as águas de lavagem apresentam valores acima desses padrões.

\section{Tratamento dos efluentes}

Precipitação alcalina dos elementos

Os resultados obtidos com este estudo indicaram que a melhor remoção dos elementos foi obtida com uma concentração de $1.180 \mathrm{mg} \mathrm{L}^{-1} \mathrm{Fe}^{3+}$ utilizando $\mathrm{Ca}(\mathrm{OH})_{2}$. Com a utilização do $\mathrm{NaOH}$, os percentuais de remoção alcançados para os três elementos estão acima de $98 \%$, porém, apenas com a utilização do $\mathrm{Ca}(\mathrm{OH})_{2}$ foi possível atender os padrões de lançamento indicados anteriormente (Brasil, 2011). Cabe ainda destacar o menor custo e maior facilidade de manuseio do $\mathrm{Ca}(\mathrm{OH})_{2}$ comparado ao $\mathrm{NaOH}$. Outro aspecto importante em relação ao efluente tratado é a diminuição de sulfato no sobrenadante com o uso $\mathrm{Ca}(\mathrm{OH})_{2}$. Esse comportamento é uma vantagem quando se deseja reaproveitar o efluente em novos processos de descontaminação da madeira, evitando o acúmulo de sulfato nas etapas de extração.

$\mathrm{O}$ pH é um parâmetro essencial na precipitação química, dessa forma, os testes foram realizados após a definição da melhor concentração do íon férrico $\left(1.180 \mathrm{mg} \mathrm{L}^{-1}\right)$. A faixa de pH investigada foi de 2 a 9 (Figura 3). A remoção completa de As (>99\%) foi obtida em pH 4, enquanto o $\mathrm{Cr}$ e o Cu foram completamente removidos do efluente a valores de $\mathrm{pH}$ superiores a 6 e 7, respectivamente. Portanto, o aumento do $\mathrm{pH}$ de 1,6 no efluente original do processo de descontaminação da madeira a pH 7, demonstrou ser uma boa opção para a remoção simultânea dos 3 elementos investigados (>99,5\%). Em relação à influência do alcalinizante, verifica-se que o $\mathrm{Ca}(\mathrm{OH})_{2}$ apresenta melhor remoção para os três elementos, em especial para $\mathrm{pH}<4$.

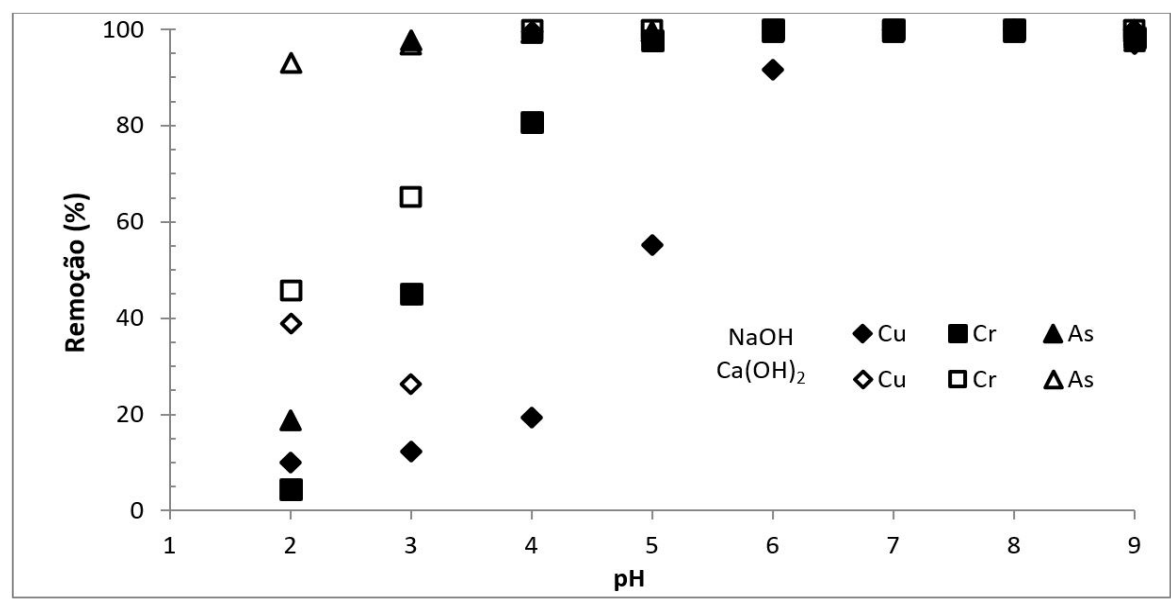

Figura 3. Percentuais de remoção de $\mathrm{Cu}, \mathrm{Cr}$ e As, durante a precipitação em diferentes $\mathrm{pH}$, do efluente resultante do processo de descontaminação da madeira utilizando $\mathrm{NaOH}$ e $\mathrm{Ca}(\mathrm{OH})_{2}$ e $1.180 \mathrm{mg} \mathrm{L}^{-1}$ de $\mathrm{Fe}^{3+}$

A Tabela 2 apresenta as condições das precipitações utilizadas e os percentuais de remoção da matéria orgânica. Salienta-se que o volume do efluente variou de 1,42 a 1,46 L, ao longo dos 7 ciclos, consequentemente, o volume de efluente reutilizado no próximo ciclo também variou. Observa-se, ainda, que houve ainda uma redução entre 30 e $62 \%$ do teor da 
matéria orgânica, indicando uma melhora na qualidade do efluente tratado em relação a esse parâmetro. A produção de lodo (resultados não apresentados) variou de 51 a $57 \%$ em relação ao volume do lixiviado obtido. Cabe destacar que quanto menor o volume de lodo gerado mais fácil é o seu gerenciamento.

Tabela 2. Remoção do carbono orgânico dissolvido (COD) e volume do efluente tratado por precipitação.

\begin{tabular}{|c|c|c|c|c|c|c|}
\hline \multirow{3}{*}{ Etapa/Ciclo } & \multirow{3}{*}{ pH } & \multicolumn{2}{|c|}{ Efluente Bruto } & \multicolumn{2}{|c|}{ Efluente Tratado } & \multirow{2}{*}{$\frac{\text { COD }}{\text { Remoção }}$} \\
\hline & & Volume & $\operatorname{COD}_{\mathrm{i}}$ & Volume & $\operatorname{COD}_{f}$ & \\
\hline & & $(m L)$ & $\left(m g C^{-1}\right)$ & $(\mathrm{mL})$ & $(\mathrm{mg} \mathrm{C} \mathrm{L-1})$ & $(\%)$ \\
\hline $\mathrm{L} 1 / \mathrm{C} 1$ & 6,99 & 1.420 & 600 & 900 & 349 & 42 \\
\hline L1/C2 & 7,12 & 1.440 & 658 & 900 & 458 & 30 \\
\hline L1/C3 & 7,11 & 1.440 & 1.310 & 900 & 768 & 41 \\
\hline $\mathrm{L} 1 / \mathrm{C} 4$ & 7,07 & 1.460 & 1.421 & 900 & 546 & 62 \\
\hline L1/C5 & 7,20 & 1.420 & 1.444 & 900 & 808 & 44 \\
\hline L1/C6 & 7,09 & 1.440 & 1.581 & 870 & 1.026 & 35 \\
\hline
\end{tabular}

Atualmente não existem estudos a respeito da reutilização do lodo proveniente do tratamento por precipitação do efluente resultante do processo de descontaminação da madeira tratada. Porém, vários estudos já foram desenvolvidos com lodos de efluentes de galvanoplastia que possuem algumas características similares como, por exemplo, a alta concentração de metais tóxicos (Espinosa \& Tenório,2000; Castanho \& Ferreira, 2001; Silva \& Castanho, 2004).

\section{Eletrorrecuperação de cobre}

Para os experimentos realizados com solução sintética de $\mathrm{Cu}\left(60 \mathrm{mg} \mathrm{L}^{-1} \mathrm{de} \mathrm{Cu}^{+2}\right.$ ) em diferentes intensidades de corrente (1 a $10 \mathrm{~A})$, a remoção percentual de Cu foi de $78 \%, 84 \%$, $90 \%, 93 \%$ e $96 \%$ para as intensidades de corrente de 1,0;2,5; 5,0; 7,5 e 10,0 A, respectivamente. Em função dos resultados obtidos, selecionou-se a corrente de $5 \mathrm{~A}$ para dar continuidade aos demais testes, por apresentar um percentual de remoção satisfatório sem a presença de depósitos negros nos eletrodos e sem aumento do potencial da célula. Foram também realizados testes com uma solução diluída de CCA $\left(\sim 60 \mathrm{mg} \mathrm{L}^{-1}\right)$, visando avaliar eventuais interferências dos demais elementos ( $\mathrm{As}$ e $\mathrm{Cr}$ ) na eletrorrecuperação do cobre. O percentual de remoção de cobre foi de $94 \%$ após 90 minutos. Mesmo com um percentual de remoção elevado (>90\%), observou-se, ao término do teste, a formação de depósitos negros nos eletrodos. Estes depósitos provavelmente devem-se à formação de $\mathrm{Cu}_{3} \mathrm{As}$ (arsenito de cobre), conforme relata Stern (2006), após análise de Difração de Raios-X para confirmar a presença de depósitos negros obtidos por deposição eletrolítica de Cu e As em uma solução de ácido sulfúrico.

Os testes com os efluentes resultantes do processo de descontaminação da madeira tratada foram realizados com efluentes em duas diferentes concentrações de $\mathrm{Cu}$, uma baixa (62 $\mathrm{mg} \mathrm{L}^{-1}$ ) e outra mais elevada $\left(166 \mathrm{mg} \mathrm{L}^{-1}\right)$, nas condições otimizadas. Ambos os testes apresentaram percentuais de remoção de Cu superiores a $90 \%$. Como esperado, uma camada levemente avermelhada foi observada sobre os eletrodos nas duas concentrações estudadas, indicando a deposição do elemento $\mathrm{Cu}$. Nesses testes não foi observado a formação de depósitos negros, sugerindo que o processo é adequado para recuperação de cobre. As concentrações dos demais elementos ( $\mathrm{Cr}$ e As), nos dois efluentes testados, apresentaram pequenos desvios, para o $\mathrm{Cr}$ de $224 \mathrm{mg} \mathrm{L}^{-1}$ a $247 \mathrm{mg} \mathrm{L}^{-1}$ e para o As de $200 \mathrm{mg} \mathrm{L}^{-1}$ a $210 \mathrm{mg} \mathrm{L}^{-1}$. Esses dados sugerem que esses elementos não influenciam no processo de eletrorrecuperação do Cu. Da mesma forma, a quantidade de COD também foi analisada nesses efluentes. Os resultados sugerem que a matéria orgânica também não influenciou na 
eletrorrecuperação de $\mathrm{Cu}$, pois durante os testes, os valores de COD não sofreram variação significativa $(\sim 3 \%)$.

Após o teste de eletrorrecuperação do $\mathrm{Cu}$, o efluente tratado foi submetido ao tratamento por precipitação utilizando as condições anteriormente otimizadas $\left(1180 \mathrm{mg} \mathrm{Fe}^{3+} \mathrm{L}^{-1} ; \mathrm{Ca}(\mathrm{OH})_{2} ; \mathrm{pH}\right.$ 7). Os percentuais de remoção atingidos após o processo de precipitação foram de $100 \%$ para As e $\mathrm{Cr}$ e de $99 \%$ para $\mathrm{Cu}$. Em seguida, as soluções foram caracterizadas e apresentaram características adequadas para descarte de acordo com os parâmetros da legislação (Brasil, 2011). Esses resultados confirmam que a aplicação sequencial da eletrorrecuperação e da precipitação química é adequada para o tratamento eficiente e mais sustentável dos efluentes da descontaminação de madeira tratada.

\section{CONCLUSÃO}

A descontaminação ácida da madeira tratada com CCA mostrou ser eficiente no processo em contracorrente para as espécies de eucalipto cultivadas no Brasil, tornando o resíduo não perigoso. A reutilização dos efluentes e das águas de lavagem reduziu significativamente o volume de efluente a ser tratado, bem como o consumo de reagentes utilizados no processo. Verificou-se ainda que a utilização das águas de lavagem não influenciou no processo de descontaminação, mantendo os percentuais de remoção estáveis.

Os tratamentos propostos para os efluentes provenientes do processo de descontaminação da madeira tratada com CCA (precipitação e eletrorrecuperação de $\mathrm{Cu}$ seguido da precipitação) foram satisfatórios. A remoção completa dos elementos investigados ( $\mathrm{Cu}$, Cr e As) pelo processo de precipitação obteve percentuais acima de $99 \%$, nas condições otimizadas em pH 7, com solução de $\mathrm{Ca}(\mathrm{OH})_{2} 100 \mathrm{~g} \mathrm{~L}^{-1}$, e agente precipitante $\mathrm{Fe}^{+3} 1.180 \mathrm{mg} \mathrm{L}^{-1}$. No processo de eletrorrecuperação de $\mathrm{Cu}$, as intensidades de corrente mais elevadas $(5,0$ a 10,0 A) proporcionaram percentuais de remoção de $\mathrm{Cu}$ superiores a $90 \%$, evidenciando que o tratamento pode ser utilizado nos extratos resultantes da lixiviação da madeira tratada com CCA.

Obteve-se um efluente adequado para o descarte de acordo com os parâmetros da legislação brasileira. Adicionalmente, a descontaminação química da madeira tratada com CCA ao término de sua vida útil é uma alternativa promissora para reduzir o impacto ambiental causado pela disposição inadequada desses resíduos perigosos.

\section{AGRADECIMENTOS}

Os autores agradecem a ANEEL e a Companhia Gaúcha de Distribuição de Energia AES Sul pelo financiamento do projeto. H. dos Santos e S. Ferrarini agradecem a CAPES pela bolsa de doutorado (Cód. financiamento 001). H. dos Santos agradece a CAPES pelo estágio de doutorado sanduíche e (PDSE n. 9108/11-4) bem como ao INRS (Centre Eau, Terre et Environnement, Canadá). M. Pires agradece ao CNPq pela bolsa PQ (312323/2015-8).

\section{REFERÊNCIAS}

Associação Brasileira de Normas Técnicas - ABNT. (2004a). NBR 10004: Classificação de Resíduos (71 p.). Rio de Janeiro: ABNT.

Associação Brasileira de Normas Técnicas - ABNT. (2004b). NBR 10005: Procedimento para Obtenção de Extrato Lixiviado de Resíduos Sólidos (3 p.). Rio de Janeiro: ABNT.

Associação Brasileira de Produtores de Florestas Plantadas - ABRAF. (2012). Anuário estatístico da ABRAF 2012: ano base 2011 (150 p.). Brasília: ABRAF.

Associação Brasileira dos Preservadores de Madeira - ABPM. (2016). Boletim Eletrônico Quinzenal, 14(659). Recuperado em 3 de outubro de 2016, de http://www.abpm.com.br

Banegas, V., Moreno, J. L., Moreno, J. I., García, C., León, G., \& Hernández, T. (2007). Composting anaerobic and aerobic sewage sludges using two proportions of sawdust. Waste Management (New York, N.Y.), 27(10), 1317-1327. PMid:17118642. http://dx.doi.org/10.1016/j.wasman.2006.09.008. 
Blais, J. F., Djedidi, Z., Cheikh, R. B., Tyagi, R. D., \& Mercier, G. (2008). Metals precipitation from effluents. Practice Periodical of Hazardous, Toxic, and Radioactive Waste Management, 12(3), 135-149. http://dx.doi.org/10.1061/(ASCE)1090-025X(2008)12:3(135).

Brasil. Conselho Nacional do Meio Ambiente - CONAMA. (2011). Resolução n. 430 de 13 de maio de 2011. Dispõe sobre a Classificação dos Corpos de Água e Padrões de Lançamento de Efluentes, complementa e altera a Resolução n. 357, de 17 de março de 2005. Diário Oficial [da] República Federativa do Brasil, Brasília.

Britto-Costa, P. H., \& Ruotolo, L. A. M. (2015). Eletrorrecuperação de cobre utilizando reator eletroquímico de leito de jorro. Quimica Nova, 38(5), 657-662.

Castanho, S. R. H. M., \& Ferreira, D. M. (2001). Incorporação de rejeitos sólidos galvânicos em vidros silicatos. In Anais do $45^{\circ}$ Congresso Brasileiro de Cerâmica, Florianópolis, Brasil. São Paulo: IPEN.

Chang, F. C., Wang, Y. N., Chen, P. J., \& Ko, C. H. (2013). Factors affecting chelating extraction of Cr, Cu, and As from CCA-treated wood. Journal of Environmental Management, 122, 42-46. PMid:23542569. http://dx.doi.org/10.1016/j.jenvman.2013.02.012.

Clausen, C. A., Kartal, S. N., \& Muehl, J. (2001). Particleboard made from remediated CCA treated wood: evaluation of panel properties. Forest Products J, 51, 61-64.

Cooper, P. A., Jeremic, D., \& Taylor, J. L. (2001). Residual CCA levels in CCA-treated poles removed from service. Forest Products J, 51(10), 58-62.

De Guardia, A., Petiot, C., \& Rogeau, S. (2007). Influence of aeration rates and biodegradability fractionation on composting kinetics. Waste Management, 28(1), 73-84.

Espinosa, D. C. R., \& Tenório, J. A. S. (2000). Laboratory study of galvanic sludge's influence on the clinkerization process. Resource, Conservation and Recycling, 31(1), 71-82.

Ferrarini, F. S. (2012). Estabelecimento de metodologia para remoção de cobre, cromo e arsênio de resíduos de madeira tratada com arseniato de cobre cromatado - CCA (Tese de doutorado). Pontifícia Universidade Católica do Rio Grande do Sul, Porto Alegre.

Ferrarini, S. F., Miranda, L. G., Maia, S. M., \& Pires, M. J. R. (2015). Madeira tratada com arseniato de cobre cromatado (CCA): opções de destino para os resíduos gerados e perspectivas no desenvolvimento de metodologias para a remoção dos elementos tóxicos. Periódico Tchê Química (Online), 12(23), 7-21.

Ferrarini, S. F., Santos, H. S., Miranda, L. G., Azevedo, C. M. N., Pires, M. J. R., \& Maia, S. M. (2012). Classificação de resíduos de madeira tratada com preservativos à base de arseniato de cobre cromatado e de boro/flúor. Quimica Nova, 35(9), 1767-1771. http://dx.doi.org/10.1590/S010040422012000900012.

Ferrarini, S. F., Santos, H. S., Miranda, L. G., Azevedo, C. M. N., Maia, S. M., \& Pires, M. (2016). Decontamination of CCA-treated eucalyptus wood waste by acid leaching. Waste Management (New York, N.Y.), 49, 253-262. PMid:26856447. http://dx.doi.org/10.1016/j.wasman.2016.01.031.

Helsen, L., \& Van Den Bulck, L. (2005). Review of disposal technologies for chromated copper arsenate (CCA) treated wood waste, with detailed analyses of thermo chemical conversion processes. Environmental Pollution, 134(2), 301-314. PMid:15589657. http://dx.doi.org/10.1016/j.envpol.2004.07.025.

Jang, Y. C., Townsend, T. G., Ward, M., \& Bitton, G. (2002). Leaching of arsenic, chromium, and copper in a contaminated soil at a wood preserving site. Bulletin of Environmental Contamination and Toxicology, 69(6), 808-816. http://dx.doi.org/10.1007/s00128-002-0132-4.

Janin, A., Blais, J. F., Mercier, G., Drogui, P., \& Kervella, H. (2009a). Selective recovery of Cr and Cu in leachate from chromated copper arsenate treated wood using chelating and acidic ion exchange resins. Journal of Hazardous Materials, 169(1-3), 1099-1105. PMid:19446391. http://dx.doi.org/10.1016/j.jhazmat.2009.04.066.

Janin, A., Zaviska, F., Drogui, P., Blais, J. F., \& Mercier, G. (2009b). Selective recovery of metals in leachate from chromated copper arsenate treated wastes using electrochemical technology and chemical precipitation. Hydrometallurgy, 96(4), 318-326. http://dx.doi.org/10.1016/j.hydromet.2008.12.002.

Janin, A., Coudert, L., Riche, P., Mercier, G., Cooper, P., \& Blais, J. F. (2011). Application of a CCA-treated wood waste decontamination process to other copper-based preservative-treated wood after disposal. Journal of Hazardous Materials, 186(2-3), 1880-1887. PMid:21216528. http://dx.doi.org/10.1016/j.jhazmat.2010.12.094.

Janin, A., Riche, P., Blais, J. F., Mercier, G., Cooper, P., \& Morris, P. (2012a). Counter-current acid leaching process for copper azole treated wood waste. Environmental Technology, 33(18), 2111-2118. PMid:23240206. http://dx.doi.org/10.1080/09593330.2012.660648. 
Janin, A., Coudert, L., Blais, J. F., Mercier, G., Drogui, P., \& Kervella, H. (2012b). CCA-treated wood waste remediation process optimization with successive recirculation loops study. Journal of Environmental Engineering, 138(2), 200-207. http://dx.doi.org/10.1061/(ASCE)EE.1943-7870.0000474.

Jekel, M., \& Amy, G. L. (2006). Interface science in drinking water treatment: theory and applications (193 p.). UK: Elsevier.

Lombardi, L. R. (2010). Análise da qualidade de moirões de eucalipto tratados comercializados em três munícipios do sul do estado do Espírito Santo (Trabalho de conclusão de curso). Departamento de Engenharia Florestal, Universidade Federal do Espírito Santo, Jerônimo Monteiro.

Santos, H. S. (2014). Remoção de elementos tóxicos em efluente proveniente da descontaminação de madeira tratada com arseniato de cobre cromatado (Tese de doutorado). Pontifícia Universidade Católica do Rio Grande do Sul, Porto Alegre.

Santos, H. S., Ferrarini, S. F., Flores, F. Q., Pires, M. J. R., Azevedo, C. M. N., Coudert, L., \& Blais, J.-F. (2018). Removal of toxic elements from wastewater generated in the decontamination of CCA-treated Eucalyptus sp. and Pinus canadense wood. J Mater Cycles Waste, 20(2), 1299-1309. http://dx.doi.org/10.1007/s10163-017-0694-1.

Silva, A. C., \& Castanho, S. R. H. M. (2004). Silicato glasses obtained from fine sílica powder modified with galvanic waste addition. Journal of Non-Crystalline Solids, 348, 211-217. http://dx.doi.org/10.1016/j.jnoncrysol.2004.08.171.

Spinelli, V. A. (2001). Quitosana: polieletrólito natural para o tratamento de água potável (Dissertação de mestrado). Universidade Federal de Santa Catarina, Florianópolis.

Stern, H. A. G. (2006). Electrolyticremediation of chromated copper arsenate wastes (Doctoral thesis). Massachussetts Institut of Technology, Boston.

Townsend, T., Dubey, B., Tolaymat, T., \& Solo-Gabriele, H. (2005). Preservative leaching from weathered CCA-treated wood. Journal of Environmental Management, 75(2), 105-113. PMid:15763153. http://dx.doi.org/10.1016/j.jenvman.2004.11.009.

Vidal, M. V., Evangelista, W. V., Silva, J. C., \& Jankowsky, I. P. (2015). Preservação de madeiras no Brasil: histórico, cenário atual e tendências. Ciência Florestal, 25(1), 257-271. http://dx.doi.org/10.5902/1980509817484.

Zagury, G. J., Dobran, S., Estrela, S., \& Deschênes, L. (2008). Inorganic arsenic speciation in soil and groundwater near in-service chromated copper arsenate-treated wood poles. Environmental Toxicology and Chemistry, 27(4), 799-807. PMid:18333683. http://dx.doi.org/10.1897/07-305.1.

Contribuição dos Autores: HSS: investigação, metodologia, validação, visualização, escrita - primeira redação e escrita revisão e edição; SFF: análise formal, visualização, escrita - primeira redação e escrita revisão e edição; MJRP: curadoria de dados, análise formal, obtenção de financiamento, administração do projeto, recursos, supervisão, visualização, escrita - revisão e edição; CMNA: análise formal, visualização, escrita - revisão e edição; LC: investigação, metodologia, validação. JFB: análise formal, administração do projeto, recursos, supervisão. 